\title{
Improving HTTP-based Video Performance using Network Flow Buffering
}

\author{
Jesse Steinberg and Joseph Pasquale \\ Dept. of Computer Science and Engineering \\ University of California, San Diego \\ La Jolla, CA, USA \\ \{jsteinbe, pasquale\}@cs.ucsd.edu
}

\begin{abstract}
We present network flow buffering, which is the use of a remote flow-regulating buffer that is deployed between a Web client and server to improve performance of HTTP-based playback of video. We show that HTTP enhanced with network flow buffering significantly improves performance, especially under high packet loss and highly variable bandwidth conditions, when compared with using either straight HTTP or streaming. The benefits of network flow buffering are numerous, and include reducing interruptions to playback, maintaining high video-image quality, and decreasing client-buffering requirements. Network flow buffering is easily implemented with existing Web mechanisms.
\end{abstract}

Keywords: Video streaming, HTTP, proxy.

\section{INTRODUCTION}

Video is an increasingly larger fraction of Internet traffic [4], with HTTP-based video streaming being very popular and supported by most video players for various reasons. One is the use of HTTP proxies from behind firewalls that allow access to the outside world. Another is that it is easier for Internet users to place video clips on Web sites provided by their ISPs as they may not have direct access to a video server, or they may lack the expertise to administer their own server. In the former case, the video can be streamed directly via HTTP, or a streaming protocol can be tunneled through HTTP. However, in the latter case, the server dictates that HTTP be used directly. When direct HTTP is used, the client cannot take advantage of specialized streaming protocols designed to optimize performance.

Network flow buffering (NFB) is a simple client-oriented approach to improving video streaming over HTTP. With NFB, an application-layer flow-regulating buffer is dynamically deployed between a Web client and server. These buffers act on a per-session basis. For example, multiple buffers can be simultaneously deployed to different locations to handle video sessions originating at different servers. Furthermore, these buffers do not need to store video data beyond the duration of a session (although they can if so desired), and in many usage scenarios will store only a small portion of the video data at any time. Dynamic deployment does require a suitable host for NFB between the client and server; however, the assumption that such a location exists is not unreasonable, considering that the location need not belong to a third party. For example, the user's own PC can be used (as in the common scenario of the user with a thin wireless client that is close to their PC at home or work).
In this paper, we compare NFB-enhanced HTTP to "direct" (standard) HTTP and to streaming in two specific scenarios. We show that the performance of NFB-enhanced HTTP is superior to both direct HTTP and streaming when there is a high rate of packet loss in a portion of the network closest to the user (e.g., a wireless access network), or when the bandwidths of that portion and the rest of the network exhibit a high degree of variation relative to each other.

The remainder of the paper is organized as follows. In Section 2, we introduce two common usage scenarios that are the focus of our investigation. In Section 3, we analyze the performance of NFB-enhanced HTTP by comparing it to direct HTTP and streaming. We review related work in Section 4, and present conclusions in Section 5.

\section{USAGE SCENARIOS}

The optimal location for NFB depends on network conditions. Ideally, NFB is located just beyond a "problem hop," generally a boundary point where there is a significant change in network performance or reliability characteristics. This location will often be at a LAN/WAN gateway, such as when the client accesses the Internet via a low-bandwidth wireless connection. Or, it may be more practically located beyond this point; perhaps the user's home or office PC may provide such a convenient location.

\section{A. Smoothing}

During a video session, the effective network bandwidth seen by the user may change as the result of changing network or server conditions. Reasons for this include changes in packet loss rate in, say, a wireless connection as the user roams, router congestion, or the server becoming overloaded and being forced to reduce the transmission rate. In this paper, smoothing refers to using NFB to mask (from the client) dynamic changes in relative bandwidth between the WAN and LAN that can cause the effective bandwidth seen by the user to be lower than the video playback rate. Thus, smoothing occurs when the WAN and LAN bandwidths are highly variable relative to each other.

Figure 1 shows a scenario of LAN and WAN bandwidths during a video session. Each line shows the potential amount of data transferred over time, and hence the slope represents the bandwidth. Although the LAN and WAN have the same average bandwidth, the WAN bandwidth is bursty while that of the LAN is constant. As shown, the effective bandwidth (i.e., minimum slope) seen by the client has a lower average. 


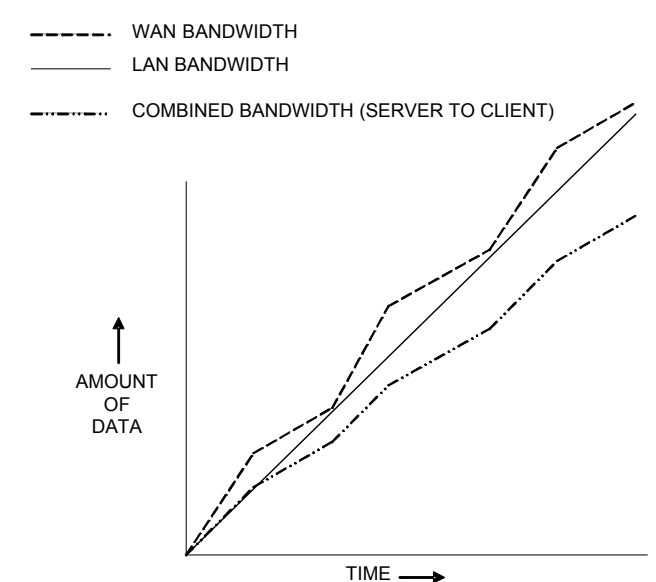

Figure 1. WAN and LAN bandwidths for smoothing scenario.

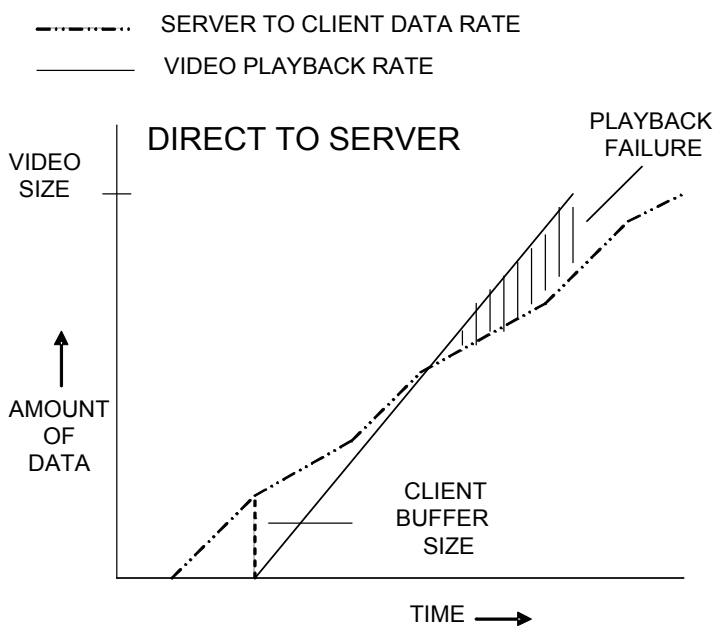

Figure 2. Failure when data rate drops below playback rate.

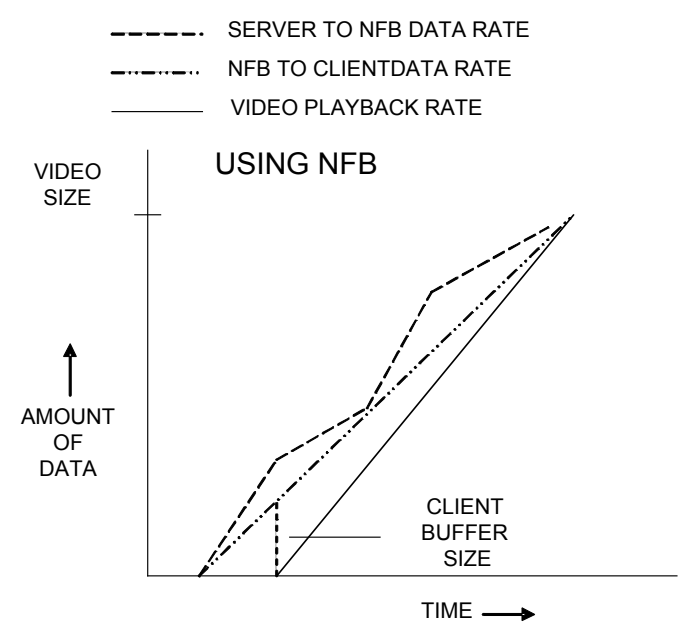

Figure 3. Benefits of smoothing with network flow buffering.

In Figures 2 and 3, video playback is depicted with and without NFB, respectively. A specialized video streaming protocol is expected to perform poorly under these conditions of high relative variability for two reasons. The effective bandwidth is considerably below the video playback rate, so the video application will be forced to either delay the video start time to buffer a large amount at the client, reduce video quality, or interrupt playback in order to buffer. Furthermore, the unpredictable nature of the bandwidth will make it more difficult for the video application to make correct protocol and buffering decisions on the fly.

When NFB is used, a smoothing effect allows the video to play smoothly. When the WAN bandwidth is higher than the LAN bandwidth, the buffer will fill up. At a later time, when the LAN bandwidth is higher than the WAN bandwidth, data will be available at the buffer in order to maintain higher throughput. In the scenario depicted in Figure 3, the client will see the bandwidth as higher than the video playback rate, and will therefore be able to maintain uninterrupted, full-quality playback. Even if the smoothed playback rate is less than the video playback rate, the frequency and duration of interruptions will still be reduced.

\section{B. Reduced Retransmission Path}

One of the primary motivations for using a specialized UDP-based video protocol is that it can selectively drop packets, thus maintaining smooth playback by reducing the frame rate or picture quality in ways that will still allow the user to have a satisfactory experience. Since HTTP uses TCP, which provides reliable transport, it does not have the luxury of selectively dropping video data, and so maintaining smooth playback is more difficult when there is packet loss. This problem is especially relevant when the user is roaming while using a wireless device, as the user may move to locations where connection quality is poor. Consequently, another benefit of having NFB between the client and server is that it reduces the path over which retransmitted packets must travel, which lessens the performance penalty incurred by packet loss.

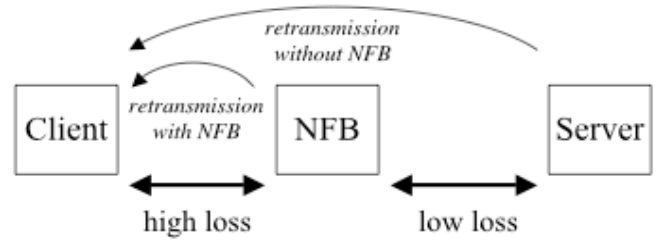

Figure 4. The packet retransmission path is reduced when using network flow buffering.

Figure 4 shows the reduced length of the retransmission paths when NFB is available. In the case of a wireless client, if the wireless access network is more prone to packet loss than the rest of the wired WAN, retransmissions will typically only have to travel between the client and intermediary supporting NFB (assuming it is beyond the wireless portion) and not suffer the delay of the entire network.

\section{PERFORMANCE COMPARISON}

We now compare NFB-enhanced HTTP to direct HTTP and to Real streaming under the two usage scenarios described in Section 2. By "Real streaming," we mean streaming as implemented by Real ${ }^{\mathrm{TM}}$ when using their 
commonly available RealOne ${ }^{\mathrm{TM}}$ Player accessing video from their servers, and essentially treating it as a black box, measuring end-to-end performance as observed by the output at the player. We chose the latter as a good representative of a video streaming protocol that is both popular and technologically mature.

\section{A. Experimental Setup}

The experimental setup is as follows. For the client, we used a notebook computer with a $500 \mathrm{MHz}$ Intel Pentium III processor running the RealOne ${ }^{\mathrm{TM}}$ Player on Windows 98. The server was a P3 933MHz PC running Windows 2000. The intermediate "gateway" machine used for NFB was a Pentium II $450 \mathrm{MHz}$ PC running FreeBSD. To simulate a network with a given bandwidth, we used DummyNet in FreeBSD (IP Firewall kernel module), which supports the creation of pipes to control bandwidth, delay, and packet loss between two communication endpoints. Four pipes are used in total, one pair for symmetric bidirectional LAN control and another for symmetric bidirectional WAN control.

\section{B. Bandwidth Variation and Smoothing}

In this experiment, we show how NFB can provide smooth playback that would otherwise be interrupted multiple times. In addition to the performance improvement, using the NFB requires no more application-layer buffering than is required when streaming directly from server to client. In fact, in circumstances where smoothing works well, it is normally expected to use less. The video clip used for this experiment was 202 seconds (3.4 minutes) in length, with a bit rate of $38.5 \mathrm{KBps}(308 \mathrm{Kbps})$.

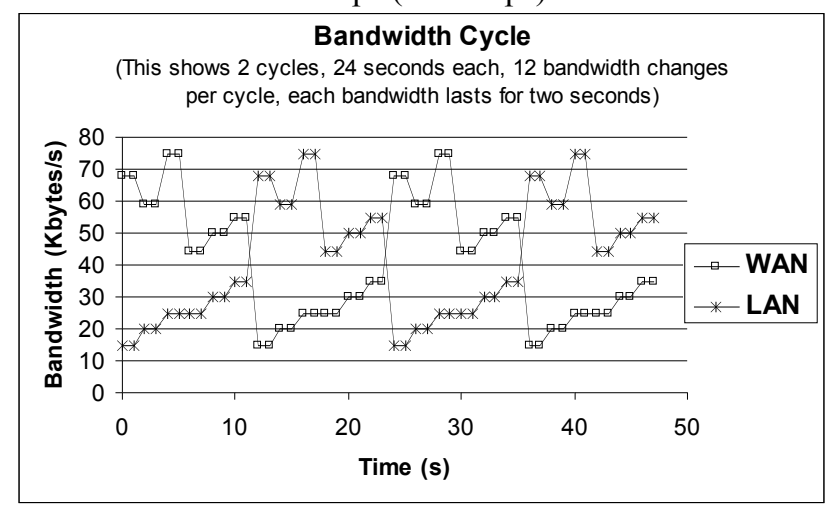

Figure 5. Bandwidth for smoothing experiment.

We used DummyNet to create cyclical client/gateway "LAN" and gateway/server "WAN" bandwidths as shown in Figure 5. The averages for the WAN and LAN for each cycle are $41.75 \mathrm{KBps}$, which is above the average video playback rate of $38.5 \mathrm{KBps}$. Without smoothing, the effective bandwidth seen by the client is the minimum at any point in time of the bandwidth cycles, averaging $25 \mathrm{KBps}$, and we expect the video player to have to interrupt playback so that the network can catch up to the video.

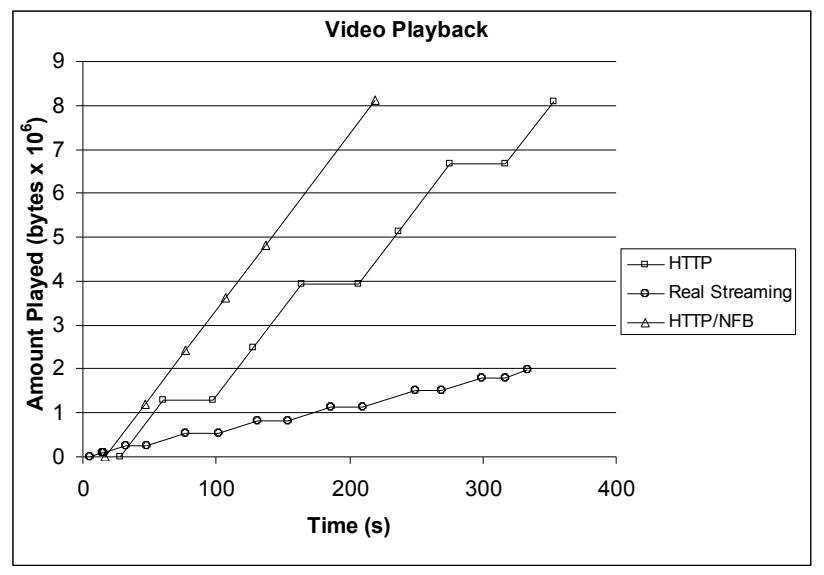

Figure 6. Playback in each of the three scenarios.

Figure 6 shows the video playback as amount of data played over time for direct HTTP, Real streaming, and NFBenhanced HTTP. With NFB-enhanced HTTP, the player buffers for 17 seconds before playback begins. Once playback begins, the video plays smoothly and at full quality for its entirety without any additional buffering by the player. This is due to the smoothing effect of NFB, which allows a higher average bandwidth to be sustained to the client. For direct HTTP (i.e., without NFB), the player buffers for 28 seconds before playback begins. During playback, the video plays at full quality, but the player interrupts the video three times to refill its buffer, for a total of 124 seconds of buffering after playback has started, and 152 seconds of total buffering, as compared to just 17 seconds with NFBenhanced HTTP.

When Real streaming is used, there is an initial 5 seconds of buffering before playback begins. The player quickly recognizes that bandwidth is inadequate and attempts to adapt accordingly. In doing so, it reduces the frame rate and picture quality, and ends up only retrieving $1.97 \mathrm{MB}$ (1966954 bytes), or about $25 \%$, of the video. This results in a choppy, "slide show" resulting in very poor-quality playback. Despite this adaptation, the video playback is still interrupted 7 times for a total of 165 seconds of additional buffering.

The extra buffering that occurs when there is no smoothing is required because the player's buffer is suffering from underflow, as the average bandwidth is lower than the video bit rate. Note that even if the player had perfect knowledge of the future, it would have to delay the start of the video by 152 seconds to ensure smooth playback under direct HTTP. Not only would this frustrate the user, it would also require a buffer size of nearly $6 \mathrm{MB}(152 \times 38.5 \mathrm{KBps})$. Given that the player cannot anticipate network traffic bursts, or that it may not be desirable to delay the start of the video for so long and force the user to wait, or to reduce memory consumption, the player is forced to interrupt the video for two periods of half a minute or more to complete playback. 


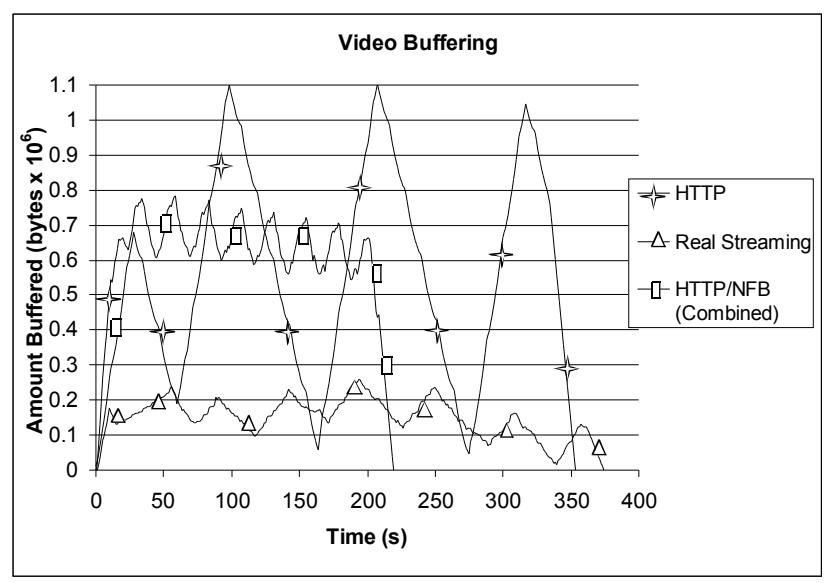

Figure 7. Buffering during the smoothing experiment.

In Figure 7, we show the amount of buffering at the player for direct HTTP, Real streaming, and NFB-enhanced HTTP (in the latter case, the combined amount buffered at both the player and intermediary for NFB is presented). The large spikes in buffering for direct HTTP result from the player trying to manage its buffer when the bandwidth is bursty. The maximum buffer size for the player in this case is $1.1 \mathrm{MB}$ (1103346 bytes). The buffering when using Real streaming is also bursty. However, the drastic reduction in amount of video data played, at the cost of playback quality, results in a maximum client buffer size of $258 \mathrm{~KB}(257805$ bytes). Finally, the smoothing effect of NFB-enhanced HTTP can be seen by the significantly reduced burstiness of its (combined) buffering.

The combined buffering when NFB-enhanced HTTP is being used is further broken down into its two components, the client buffering and the intermediary (for NFB) buffering, in Figure 8 . When the intermediary buffer is peaking because the WAN bandwidth is higher than that of the LAN, the client buffer is draining because the LAN bandwidth is lower than the video rate. When the LAN bandwidth increases, the client buffer will start to increase, but the intermediary buffer begins to drain since the WAN is now the bottleneck. The maximum combined buffering (client + intermediary) peaks at $785 \mathrm{~KB}$ ( 785057 bytes), less than the maximum buffer requirement at the client when direct HTTP is used. The maximum buffering at the client when the NFB is used is $660 \mathrm{~KB}$ (660514 bytes). This is due to the fact that without NFB, the average effective bandwidth over the course of the playback is lower, so more buffering is required to compensate.

\section{Packet Loss}

In this experiment, we show how NFB reduces retransmission delay under packet loss for HTTP streaming, which can result in video performance comparable to that of Real streaming. Here, we configured DummyNet to randomly drop packets in the LAN portion, to compare how direct HTTP, Real streaming, and NFB-enhanced HTTP tolerate packet loss.

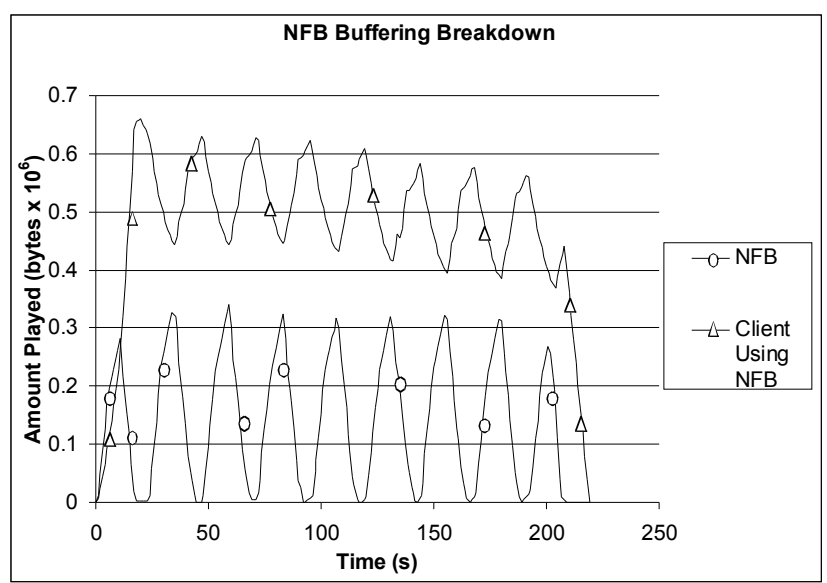

Figure 8. Breakdown of buffering at client and NFB.

The random loss of packets is expected to reduce the effective bandwidth. However, under TCP, the limited latency between the client and NFB-intermediary allows for a higher tolerance of packet loss than under the full end-toend latency of the entire network, since it will take less time to retransmit lost packets. (In our experiments, the LAN delay was $10 \mathrm{~ms}$, and the WAN delay $50 \mathrm{~ms}$, for a total endto-end delay of $60 \mathrm{~ms}$.) When Real streaming is used, the full latency is still a factor, but the protocol may selectively choose to allow packets to be lost rather than retransmitting. Thus, Real streaming, if it adapts well, is expected to avoid video interruptions at the cost of reduced playback quality.

We ran three trials, each having DummyNet configured with a different packet loss rate which remains constant throughout the course of the trial. We chose packet loss rates of $2 \%, 4.5 \%$, and $5 \%$ because each is a threshold where we observed that one of the scenarios typically starts to encounter interruptions to playback. The $2 \%$ rate was chosen because it was the threshold where direct HTTP started to degrade in performance and was no longer competitive with the other approaches (interruptions in playback occur at this loss rate). The $4.5 \%$ loss rate is the corresponding threshold for Real streaming; interruptions in playback start occurring at this point. Finally, at 5\% loss rate we see interruptions in playback even with the NFB approach.

We expect direct HTTP to be the least tolerant of packet loss. Figure 9 shows the playback under 2\% packet loss for each scenario. With direct HTTP, there is a 20 second interval of buffering before the playback starts, and there is an additional 29 second interruption in playback. Neither of the other two scenarios incurs an interruption during playback. With NFB-enhanced HTTP, there is an initial 14second buffering period before playback begins, after which the video plays at full quality without interruption. When Real streaming is used, the video takes the longest to start, as there is an initial 28 -second buffering period. The video then plays at nearly full quality without interruption, with about $95 \%$ of the video data being played. As the packet loss rate increases and the effective bandwidth over the network (with a $60 \mathrm{~ms}$ delay) decreases, we expect direct HTTP playback to 
continue to degrade in the form of increased frequency and duration of interruptions of video playback for re-buffering.

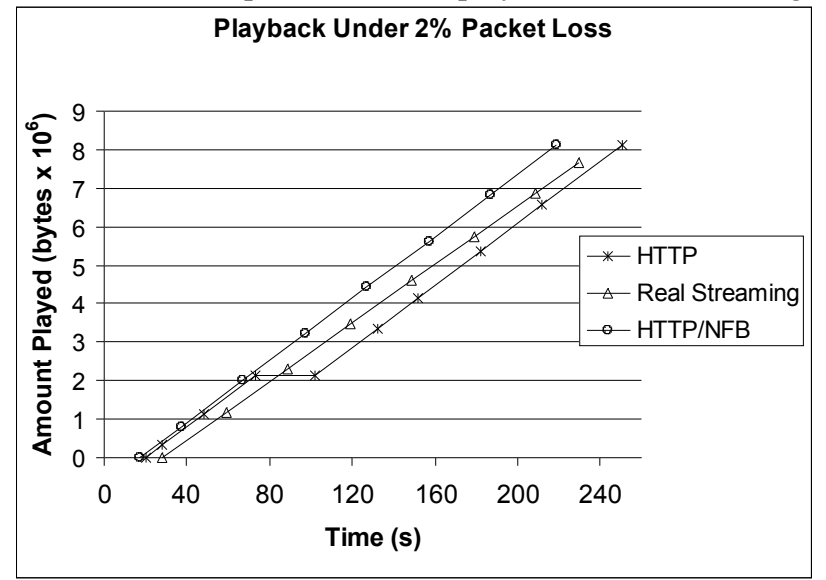

Figure 9. Playback under 2\% packet loss when using Real streaming vs. network flow buffering.

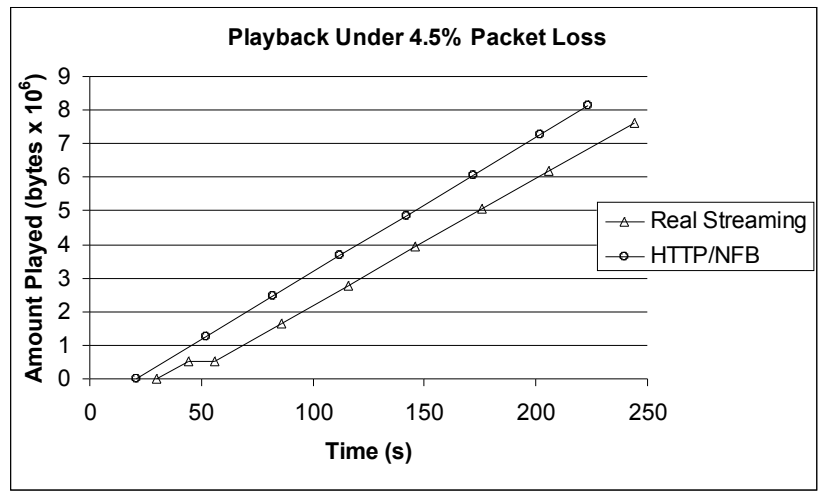

Figure 10. Playback under $4.5 \%$ packet loss when Real streaming vs. NFBenhanced HTTP.

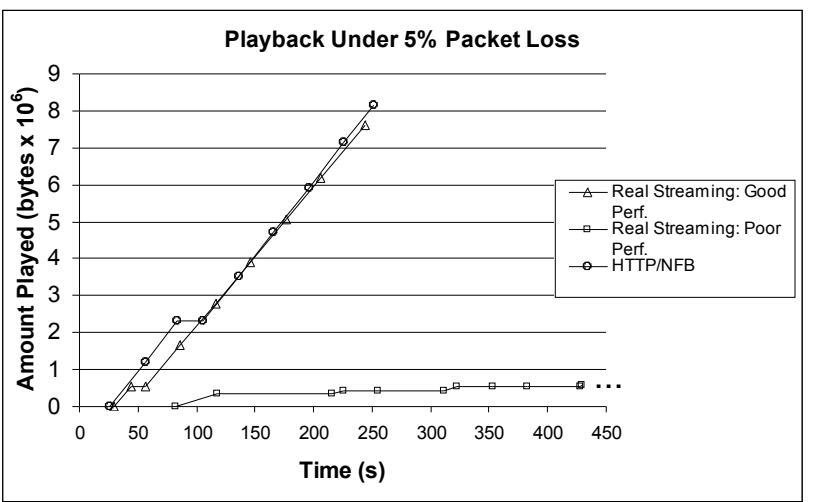

Figure 11. Playback under 5\% packet loss when using Real streaming vs. NFB-enhanced HTTP.

We now compare how NFB-enhanced HTTP and Real streaming perform at higher packet loss rates. Figure 10 shows the playback with a $4.5 \%$ packet loss rate. With Real streaming, there is an initial buffering period of 30 seconds before playback begins, and an additional interruption of 12 seconds to buffer more data that occurs 14 seconds into the playback. Up until this point, the playback is very choppy, with only between 1 and 4 frames displayed per second and many artifacts on the screen. However, after this interruption, the playback smoothens out, though the frame rate does occasionally drop to $2 / 3$ its normal rate. With NFB-enhanced HTTP, there is an initial buffering period of 21 seconds before full-quality uninterrupted playback begins. Both protocols allow smooth playback when the packet loss rate is more than double what direct HTTP can tolerate. The amount of time playback is delayed or suspended for buffering using NFB-enhanced HTTP is roughly half that when using Real streaming.

Finally, we examine what happens with a packet loss rate of $5 \%$, where we begin to see interruptions during playback when NFB-enhanced HTTP is used, and erratic behavior from Real streaming. This case is shown in Figure 11. When the packet loss rate is $5 \%$, Real streaming exhibited mixed behavior. About half of the trials, it would adapt well to the packet loss, resulting in smooth playback at slightly reduced quality. But the other half of the trials, it would not adapt well, resulting in frequent interruptions in playback, where each interruption might last for 1 to 2 minutes!

The poor performance example of Real streaming, which is cut off to fit into the chart, continues the same trend of frequent, long interruptions for the entire video, and playback can last 20 minutes or more. When Real streaming adapts well, it buffers for about 12 seconds before playback begins, and has one buffering interruption of 18 seconds about 6 seconds into the playback.

When NFB-enhanced HTTP is used, there is an initial buffering period of 26 seconds before playback begins. About 57 seconds into the playback, there is an interruption that lasts for about 23 seconds. Thus, the general behavior of NFB-enhanced HTTP is similar to Real streaming when the latter is operating well.

As the packet loss rate rises above 5\%, we expect an increase in the frequency and duration of interruptions to playback, and that Real streaming will be less able to adapt and will more frequently exhibit the behavior of the poor playback case.

\section{Startup Delay Considerations}

NFB does not necessarily pre-buffer any data before streaming it to the client. In fact, in the smoothing and packet loss experiments described previously, data is only buffered passively, when the NFB-to-Client bandwidth is less than the Server-to-NFB bandwidth. In these scenarios there is no initial buffering delay at the NFB host.

If the NFB location is chosen to optimize video playback from a particular server, only the first video played from this server will incur any deployment overhead. All subsequent sessions can use the same NFB instance. If the location is chosen to optimize a problematic network link such as the wired/wireless boundary (e.g. the user's desktop PC or the wireless/wired gateway), the same NFB instance can be used for all video sessions after the initial deployment.

Our experiments demonstrated that in the packet loss scenarios, the use of NFB reduced the initial client buffering before playback begins. In the experiment using a $4.5 \%$ 
packet loss rate, the video started 9 seconds faster when using NFB as compared to when using Real streaming despite that fact that Real adjusted the playback quality to compensate for the high loss rate. In all of our experiments, the video starts sooner with NFB than with direct HTTP.

In the smoothing scenario, using Real streaming improves the video start time at the cost of a reduction in quality. In our experiments, the reduction in quality was drastic; the actual reduction in quality will depend on the network conditions at the time the video is played. NFB is primarily designed for situations in which HTTP must be used due to server or client limitations. However, if the video server supports a streaming protocol, and the client has access to the protocol (e.g., the client is not behind a restrictive firewall), a good compromise is to start playback using the streaming protocol, and give the user the option to switch to HTTP with NFB if the user is dissatisfied with the reduced quality and is willing to wait for a restart.

\section{RELATED WORK}

There is a large body of research on smoothing compressed video streams by sending frames to the client buffer ahead of their playback times to deal with bandwidth burstiness. In this approach, a video transmission schedule is calculated at the server based on the bit rate over the course of the video, the server bandwidth, and the amount client memory available for buffering. In [5], the smoothing takes into account the possibility of having multiple intermediary buffers (such as proxies) along the path between server and client, and considers bandwidth limitations of network hops among the intermediary buffers, the client, and the server. In [3], the constraint of minimizing peak server bandwidth is relaxed to keep the client and server in closer synchronization, which better supports interactive functionality such as fast-forward and rewind. Optimal smoothing can be approximated for live video by calculating the transmission schedule over a window of time based on the currently available video data in conjunction with predictive techniques [7]. We are attempting to solve a different problem, of dynamic changes in network performance and availability as a result of such factors as wireless network variability and congestion, for HTTP streaming. Our approach is transparent to the server so it does not require existing servers to change.

Video performance can also be improved using proxy caching. Some approaches perform partial caching of video streams to improve performance, while reducing resource consumption at the proxy $[6,8,9,10]$. Other approaches use cooperative caches to improve hit rates as well as reduce server load and WAN bandwidth utilization $[1,2,11]$. There are a number of important differences between NFB and proxy caching. In proxy caching, videos are cached at a shared server accessed by many clients, so resources must be shared and the proxy can become a bottleneck. NFB is a client-specific approach, so there is no competition for resources among multiple users. Furthermore, the location of a shared proxy cache may not be ideal for a particular user. NFB can operate under dynamic deployment, and the location can be chosen to best serve one particular client.

\section{CONCLUSIONS}

In this paper, we demonstrated that network flow buffering could make HTTP-based video competitive with, and in some ways even better than, Real streaming, under the conditions of high LAN packet loss rates or in networks with highly variable relative LAN/WAN bandwidths. Our experiments showed that the smoothing benefits of NFBenhanced HTTP support full-quality playback, while requiring less client buffering than direct HTTP, in cases where Real streaming will reduce buffering requirements at the cost of drastically reduced playback quality.

The ability to dynamically deploy a simple flowregulating buffer has the added benefit of reducing retransmission delay in order to increase playback performance under high LAN packet loss rates. In our experiments, using NFB-enhanced HTTP allows the video player to tolerate more than double the packet loss rate that direct HTTP can tolerate. The lower the delay is between the NFB-intermediary and the client, the more tolerant the streaming will be to packet loss. Furthermore, using NFBenhanced HTTP outperforms Real streaming at medium packet loss rates, and is at least competitive with Real streaming at higher loss rates where Real streaming begins to exhibit erratic behavior.

\section{REFERENCES}

[1] S. Acharya and B. Smith, "MiddleMan: A Video Caching Proxy Server," Proc. 10th NOSSDAV, June 2000.

[2] M. Dahlin, R. Wang, T. Anderson, D. Patterson, "Cooperative Caching: Using Remote Client Memory to Improve File System Performance," Proc. OSDI, Nov. 1994.

[3] W. Feng, "Rate-Constrained Bandwidth Smoothing for the Delivery of Stored Video," Proc. IS\&T/SPIE Multi-media Networking and Computing, Feb. 1997.

[4] Hitwise Press Report, "Hitwise Data Shows Overall Visits to Video Search Sites Up 164\%," http://www.hitwise.com/presscenter/hitwiseHS2004/videosearch.php, May 24, 2006.

[5] J. Rexford, and D. Towsley, "Smoothing Variable-bit-rate Video in an Internetwork," IEEE/ACM Trans. Networking, pp. 202-215, Apr. 1999.

[6] S. Sen, J. Rexford, and D. Towsley, "Proxy Prefix Caching for Multimedia Streams," Proc. INFOCOM, Mar. 1999.

[7] S. Sen, J. Rexford, J. Dey, J. Kurose, and D. Towsley, "Online Smoothing of Variable-bit-rate Streaming Video," IEEE Trans. Multimedia, pp. 37-48, Mar. 2000.

[8] Y. Wang, Z. Zhang, D. H.C. Du, and D. Su, "A Network Conscious Approach to End-to-End Video Delivery over Wide Area Networks Using Proxy Servers," Proc. IEEE INFOCOM, Apr. 1998.

[9] K.-L. Wu, P. S. Yu, and J. L. Wolf, "Segment-based Proxy Caching of Multimedia Streams," Proc. WWW, 2001.

[10] [S. Jin, A. Bestavros, A. Iyengar, "Network-Aware Partial Caching for Internet Streaming Media Delivery," ACM/Springer Multimedia Systems Journal, vol. 9, no. 4, Springer-Verlag, Oct. 2003.

[11] D. A. Tran, K. A. Hua, and S. Sheu, "A New Caching Architecture for Efficient Video Services on the Internet," Proc. IEEE Symp. Applications and the Internet (SAINT), Jan. 2003. 